\title{
SIMULATION-BASED CONTROL FOR GREEN TRANSPORTATION WITH HIGH DELIVERY SERVICE
}

\author{
Seokgi Lee \\ Vittaldas V. Prabhu \\ Pennsylvania State University \\ Department of Industrial and Manufacturing Engineering \\ University Park, PA. 16802 USA
}

\begin{abstract}
Shipping operations are facing increasing pressures for tighter delivery service levels and green transportation, which conflict with each other and requires trade-off between fuel consumption and delivery service. Furthermore, responsiveness to customer demand requires rapid generation of good quality solutions. This paper presents a simulation-based feedback control algorithm for real-time vehicle route planning which considers delivery timeliness and fuel efficiency. The proposed control theoretic algorithm uses feedback from simulation to adjust the planned routes for timeliness and adaptively adjust the vehicle speed within an allowable range to improve fuel efficiency. The formulation results in a multivariable continuous variable control system with non-linear dynamics. The control algorithm extends prior work in distributed arrival time control, which is used as a basis to derive analytical insights into this computational intractable optimization problem. Performance of the algorithm is evaluated using a simulation model of an industrial distribution center.
\end{abstract}

\section{INTRODUCTION}

Transportation is the most expensive logistics activity in terms of not only the transportation cost itself but also its ripple effects on the rests of processes which are sensitively affected by expected delivery time. Especially for the time constraint which is considered significantly for a variety of areas such as purchasing, inventory and customer delivery, on-time delivery has played an important role in improving the rest of processes and services. Many companies and organizations including manufacturing companies, third-party logistics providers, and various service organizations have tried to improve their service ability to meet their customer requirements against specifications for delivery time. While transportation costs have always been a key determinate in terms of selecting manufacturers and service providers, the emphasis on timely delivery is becoming increasingly important for both suppliers and consumers.

The performance measure of on-time delivery is percent achievement of specific delivery time or time window demanded by customers or the post processes. This time metric is used to improve service quality along with decreasing transportation costs through systematic barrier removal. In a traditional manner for managing and operating transportation, delivery sequences considering both cost and time metrics are decided before dispatching vehicles. With growing sophisticated customer demands, the organizations and companies are under pressure to improve their transportation strategies and methods to satisfy these demands so that they can improve both service levels and fuel consumption. One of the transportation issues affecting operational performances is unexpected new demands or demand changes. In particular, if the vehicle is already operating with given delivery sequences, unexpected new demands or demand 


\section{Lee and Prabhu}

changes are very difficult to be reflected on the existing sequences. They should be applied in real-time to maintain overall transportation performance and guarantee minimum exchange costs.

In terms of algorithm performance for transportation scheduling, metaheuristic or simulation methods rather than optimization approaches are usually used to reflect demand changes into existing solutions due to computational complexity. The important issue of heuristic or metaheuristic for the vehicle routing scheduling is to find appropriate methods to diversify search space and intensify routing solution to reduce both transportation cost and time gap from demands. Along with these common approaches, control theoretic approaches based on discrete event simulation have been developed recently. One of the most important benefits of this approach, in terms of algorithm performance, is quick convergence into a relatively good solution and this property provides significant benefits to highly changeable transportation environment. Cho and Prabhu (2007) developed a unified control system using a continuous controltheoretic approach for distributed production scheduling in highly autonomous manufacturing environment. They considered multi-criteria objectives such as production rate and due date deviations and developed distributed controller to manage both job scheduling and machine capacity simultaneously. Tamani, Boukezzoula, and Habchi (2009) developed the simulation model using continuous control approach for machine capacity allocation and real-time scheduling. These control theoretic approaches can be applied to the transportation problem due to the similarities of the performance measures and problem environments.

In this paper, a unified control algorithm for both distributed arrival time control (DATC) and distributed vehicle speed control (DVSC) based on the control theoretic approach is proposed to find efficient ways to satisfy not only less transportation cost but also a better customer service level. Core of the unified control algorithm for both vehicle arrival time and speed control is constructed based on DATC which is a feedback control-based scheduling approach that attempts to minimize the average of the square of the due-date deviation (MSD) for Just-in-Time system. Analytic models for the unified control model are developed and compared with simulation results. Furthermore, the relationship between MSD and corresponding vehicle fuel efficiency is provided so that transportation managers can obtain an insight for their transportation management strategy considering both customer service improvement and cost reduction.

\section{OVERVIEW OF DATC}

Distributed arrival time control (DATC) is a closed-loop distributed control algorithm for scheduling problems which have time-criteria such as manufacturing shop floor scheduling and vehicle routing problems. For the manufacturing shop floor case, each part controller uses only its local information, such as parts arrival time, processing time and due-date, to minimize deviation from its part's due-date (Prabhu and Duffie 1999). It is effective for a highly autonomous manufacturing environment in which the scheduling algorithm should respond to processing orders which have quick processing demands and unpredictable order changes. It is performed using virtual machines with virtual parts and finally releases physical parts into physical shop-floor based on the simulation result. Because the scheduling and part releasing procedures should be performed continuously in response to real production events, computational time is one of the important performance measures to verify the scheduling algorithm.

Similarly, DATC is used in a vehicle routing problem, especially focusing on satisfying customers' due-date constraints. Like the manufacturing case, DATC uses its local information including arrival time of customer orders, transport time and due-date demands by customers to minimize deviations from duedate demanded by the customers (Lee and Prabhu 2009). It is more powerful when the order changes arise frequently. Fast convergence into good solution spaces makes flexible schedule changes possible for decision makers and, consequently, improves overall operational performance in terms of costs and service quality.

To explain the DATC structure, we assume that DATC is applied for vehicle routing scheduling. In the DATC approach, feedback control is used as a mechanism to compensate for the lack of global information by which order entities iteratively adjust the times at which they request vehicle(s) required for 


\section{Lee and Prabhu}

transportation as illustrated in Figure 1. Here, the time at which a customer order requests a vehicle is called the arrival time of the customer order at that vehicle. In this system, each controller iteratively adjusts its arrival time in order to be completed at its due-date based on feedback of completion time by the following control equation.

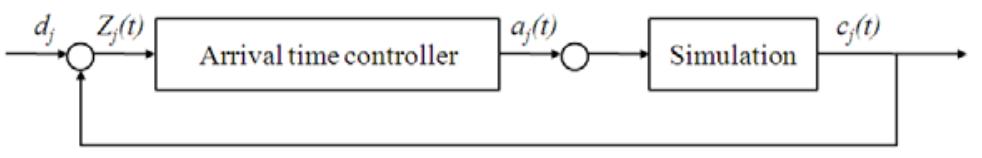

Figure 1: Closed-loop control structure for DATC approach

$$
a_{j}(t)=k_{j} \int_{0}^{t}\left(d_{j}-c_{j}(\tau)\right) d \tau+a_{j}(0)
$$

where $k_{j}$ is the controller gain, $a_{j}(0)$ is the arbitrary initial arrival time, $d_{j}$ is the due-date and $c_{j}(\tau)$ is the predicted order completion time for the $j$ th customer order in the system. For the performance measure, DATC uses mean-squared due-date deviation (MSD) which measures sum of differences between customer orders' due-date and their completion times and has the following form

$$
\text { MSD }=\sqrt{\frac{\sum_{j=1}^{n}\left(d_{j}-c_{j}\right)^{2}}{n}}
$$

where, for the order $j, d_{j}$ is the due-date, $c_{j}$ is order completion time and $n$ is the total number of orders.

One of the important characteristics of DATC is the relationship between arrival time dynamics and scheduling system conditions. In DATC system, when their due-date cannot be made, the arrival times continuously change their relative sequences within small boundary area. Figure 2 illustrates the arrival time trajectories of 3 -orders scheduling example which has initial arrival time $[10,50,100]$, transport time $[22,15,79]$, and due-date $[156,173,165]$, and its corresponding order completion time and MSD trajectories when the due-dates of each order are infeasible. Each of horizontal axes indicates simulation time (iterations) and the vertical axes are arrival time, completion time and MSD values, respectively. After some iterations, DATC system chatters and exhibits sliding modes in the steady-state where arrival times are nearly equal and oscillate in a boundary layer (Cho and Prabhu 2002). Such oscillation changes the relative order of arrival times leading to various order-transport sequences. The arrival time vector consists of the orders which have infeasible due-dates. It converges in a point and oscillates with an infinitesimal amount of arrival time vector changes during DATC simulation. When the arrival time vector shows this behavior, the infinitesimal changes of the arrival time vector in the arrival time space create $n$ ! completion time vectors and these are matched with $n$ ! transporting-sequences as described in Figure 3. The completion time vectors are located in a hyper-plane including the due-date vector for the 3-orders case.

\section{UNIFIED MODEL FOR DISTRIBUTED ARRIVAL TIME AND VEHICLE SPEED CONTROL}

The objective of the unified model consisting of distributed arrival time and vehicle speed control is to make a vehicle routing schedule by simultaneously adjusting relative orders of each demand's arrival time and vehicle speed to minimize due-date deviation. For this, the processing time of DATC is modified using the following mechanisms. 


\section{Lee and Prabhu}

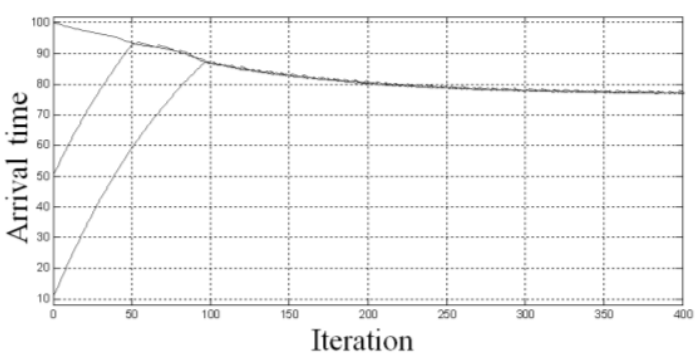

(a) Arrival time

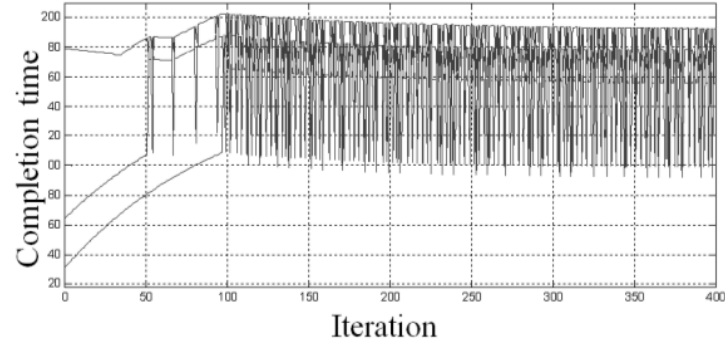

(b) Completion time

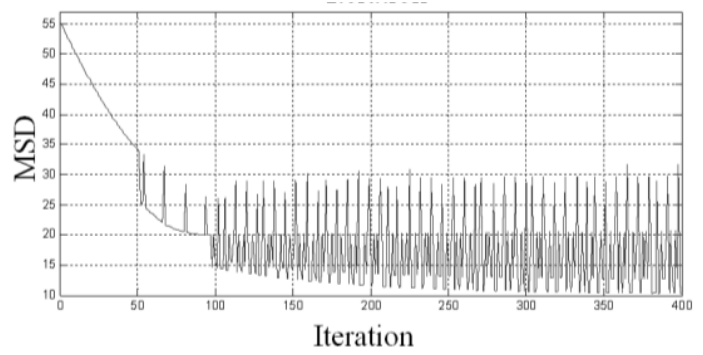

(c) MSD

Figure 2: Arrival time trajectory and corresponding results (3-orders and infeasible due-date case)

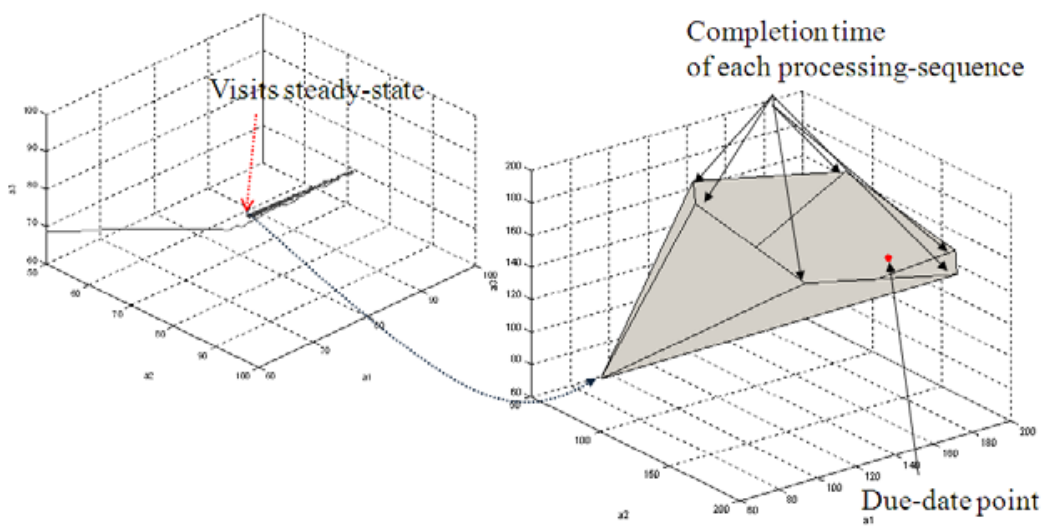

Figure 3: Convex hull geometry of arrival time

If $j$ th customer order has regular transport time $p_{j}$ which is normally determined by road speed limit, and previous order completion time is $c_{j-1}$, then the possible driving time to meet the $j$ th customer order is $d_{j}-c_{j-1}$ which is called slack time for $j$ th order. The vehicle speed control is needed when the $j$ th order cannot be made with the regular vehicle operation. During the simulation, the regular vehicle speed between specific sections should be controlled so that the regular transport time $p_{j}$ can be adjusted to meet the delivery time $d_{j}$ demanded by a customer. Transport time from $(j-1)$ th customer to $j$ th customer in the $n$th routing-sequence out of $N$ ! routing-sequences is represented as $p_{j}^{n}$. Because transport time for specific customer order is identical based on each routing-sequence, $p_{j}^{n}$ has the following property:

$$
p_{j}^{1} \neq p_{j}^{2} \neq \cdots \neq p_{j}^{n} \neq \cdots \neq p_{j}^{N !} .
$$




\section{Lee and Prabhu}

If we represent regular transport time which is applied by the distributed speed control as $\psi$, it is calculated by the following conditions which are basically based on slack time between customer orders,

$$
\psi_{j}^{n}(t)=\left\{\begin{aligned}
& \alpha_{j}^{e} p_{j}^{n}(t), \text { if } p_{j}^{n} \leq d_{j}-c_{j-1} \text { and } d_{j}-c_{j-1}>0 \\
& \alpha^{\max } p_{j}^{n}(t), \text { if } d_{j}-c_{j-1}<0 \\
& \alpha_{j}^{t} p_{j}^{n}(t), \text { if } p_{j}^{n}>d_{j}-c_{j-1} \text { and } d_{j}-c_{j-1}>0
\end{aligned}\right.
$$

where $\alpha_{j}^{e}$, is the proportional factor for the cases of earliness for the $j$ th order, $\alpha_{j}^{t}$ is the proportional factor for the case of tardiness for the $j$ th order, and $\alpha^{\max }$ is the maximum proportional factor for the case of excess $(j-1)$ th completion time with $j$ th due-date time. The proportional factor, $\alpha_{j}^{\theta}$ and $\alpha_{j}^{t}$ are bounded by following conditions:

$$
\begin{gathered}
1<\alpha_{j}^{e}<\frac{c_{r \theta g}+\delta_{j}}{c_{r \theta g}} \leq \alpha^{\max } \\
\alpha^{\min } \leq \frac{c_{r \theta g}-\delta_{j}}{c_{r \varepsilon g}}<\alpha_{j}^{t}<1,
\end{gathered}
$$

where $C_{r e g}$ is regular vehicle speed to maintain regular transport time $p_{j}(t), \alpha^{\min }$ is the minimum proportional factor, and $\delta_{j}$ is a positive value, respectively. Likewise property of $p_{j}^{n}, \psi_{j}^{n}$ has the following property:

$$
\psi_{j}^{1} \neq \psi_{j}^{2} \neq \cdots \neq \psi_{j}^{n} \neq \cdots \neq \psi_{j}^{N !}
$$

The distributed vehicle arrival time and speed control using the equation (4) is redefined from the original DATC of equation (1) as follows:

$$
a_{j}(t)=k_{j} \int_{0}^{t}\left\{d_{j}-\psi_{j}^{n}(\tau)\right\} d \tau+a_{j}(0)
$$

Using this integral controller, DATC algorithm continuously calculates due-date deviation and adjusts relative arrival time during the pre-defined number of simulation time. These adjustments create system dynamics which are identified by three regions as described in the following definition (Prabhu 2000).

Definition 1. On the arrival time vector space, three regions exist according to the position of due-date point. 1) Decoupled region: all the queuing times are zero and there is no interaction among customer orders, 2) Dead-zone region: all customer orders bar the first order in the fragment incur a queuing time, leading to interaction among two or more orders, 3) Discontinuous region: all customer orders bar the first order in the fragment incur a queuing time, leading to interaction among two or more orders.

The system dynamics in the decoupled region is explained by

$$
\dot{a}_{j}(t)=k_{j}\left(d_{j}-a_{j}(t)-\psi_{j}^{n}\right)
$$

where $p_{j}^{l}$ is the transport time between $(j-1)$ th order and the $j$ th order in the $l$ th routing-sequence. In this region, there is no queuing time, that is, the algorithm could provide just-in-time delivery. The resulting arrival time follows the following equation: 


$$
a_{j}(t)=\left(d_{j}-p_{j}^{l}\right)\left(1-e^{-k_{j} t}\right)+a_{j}(0) e^{-k_{j} t},
$$

The dynamic of the $j$ th order of the $l$ th routing-sequence in the dead-zone region is

$$
\dot{a}_{q_{j}}(t)=k_{q_{j}}\left\{d_{q_{j}}-a_{q_{1}}(t)-\left(\psi_{q_{1}}^{n}+\psi_{q_{2}}^{n}+\cdots+\psi_{q_{j}}^{n}\right)\right\}
$$

where the subscript $q_{j}$ is the customer order in the $j$ th position. The arrival time in the dead-zone region is derived as

$$
\begin{aligned}
a_{q_{j}}(t)= & \frac{k_{q_{j}}}{k_{q_{1}}}\left(d_{q_{1}}-\psi_{q_{1}}^{n}\right)\left(1-e^{-k_{q_{1}} t}\right)-\frac{k_{q_{j}}}{k_{q_{1}}} a_{q_{1}}(0)\left(1-e^{-k_{q_{1}} t}\right) \\
& +k_{q_{j}}\left(d_{q_{2}}-d_{q_{1}}-\psi_{q_{2}}^{n}-\cdots-\psi_{q_{j}}^{n}\right) t+a_{q_{j}}(0) .
\end{aligned}
$$

The arrival time dynamic in the discontinuous region is

$$
\dot{a}(t)=k(\alpha-a(t))
$$

where

$$
\begin{gathered}
k=\frac{\psi_{1}^{n}+\psi_{2}^{n}+\cdots+\psi_{N}^{n}}{\psi_{1}^{n} / k_{1}+\psi_{2}^{n} / k_{2}+\cdots+\psi_{N}^{n} / k_{N}}, \\
\alpha=\frac{\psi^{T}(d-r)}{\psi_{1}^{n}+\psi_{2}^{n}+\cdots+\psi_{N}^{n}},
\end{gathered}
$$

Here, bold $\psi, \boldsymbol{d}$, and $\boldsymbol{r}$ mean the delivery time vector, the due-date vector, the disturbance vector, respectively. Each vector has the forms, $\psi=\left[\psi_{1}^{n}, \psi_{2}^{n}, \ldots, \psi_{N}^{n}\right], \quad d=\left[d_{1}, d_{2}, \ldots, d_{N}\right]$ and $r=\left[\left(q_{1}+\psi_{1}^{n}\right),\left(q_{2}+\psi_{2}^{n}\right), \ldots,\left(q_{n}+\psi_{N}^{n}\right)\right]$, respectively. The resulting arrival time in the discontinuous region is formulated as

$$
a(t)=\alpha\left(1-e^{-k t}\right)+a(t) e^{-k t}
$$

for all customer orders.

To evaluate the analytic models for each dynamic region of the unified controller, we compare simulation results using equation (8) with analytic solution of equation (10), (12), and (16) using a problem set in which a new customer order occurs before completing previous orders. The initial problem set consists of two customer orders which have due-date 10 and 8, respectively as shown in Table 1. After 250 iterations, a new customer order which has due-date 7 occurs. Along with this new customer order, due-date of the first order is changed from 10 to 8 . Based on these changes, dynamics of arrival times for feasible and infeasible customer orders can be observed and the relative orders of arrival time of each customer order are used to decide vehicle routing sequences. We set transport time $\boldsymbol{p}=[1,2,3]$, initial arrival time $\boldsymbol{a}=[0.1,0.2,0.3]$, and the control gain $k=0.1$ for all customer orders. Although transport time for each customer could be different according to the previous customer's location, we assume that it has a value corresponding to the destination regardless of previous locations. For the speed control, we set maximum vehicle speed as regular transport time $\boldsymbol{p}$ multiplied by 1.2 which is the proportional factor when earliness occurs. On the other hand, if tardiness occurs, $\boldsymbol{p}$ is adjusted by multiplying 0.8 for each transport time. Figure 4 (a) shows arrival time trajectories of three orders from simulation using equation (8). Compared to the simulation result, analytical solution shows almost similar patterns of arrival time trajectories which are exponentially converged as illustrated in Figure 4 (b). 


\section{Lee and Prabhu}

Table 1: Customer orders and due-date variation

\begin{tabular}{|c|c|c|c|c|}
\hline Iteration & Order 1 & Order 2 & Order 3 & Due date condition \\
\hline $0 \sim 250$ & 10 & 8 & - & All feasible and distinct \\
\hline $250 \sim 400$ & 8 & 8 & 7 & Some infeasible and equal \\
\hline
\end{tabular}

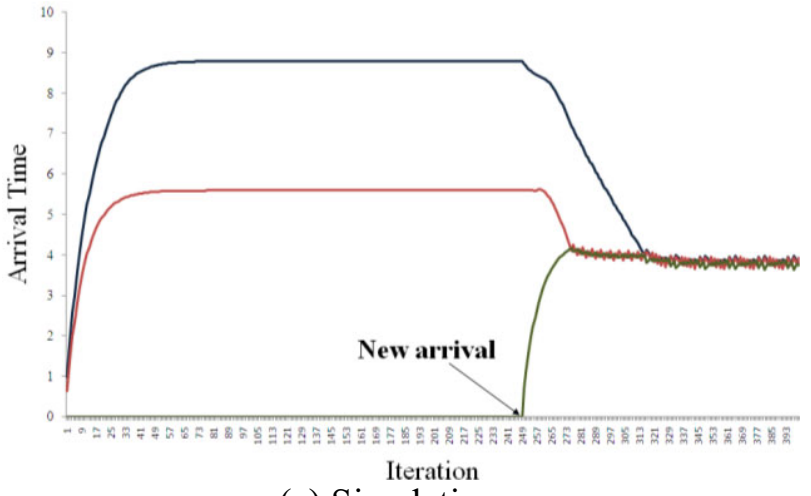

(a) Simulation

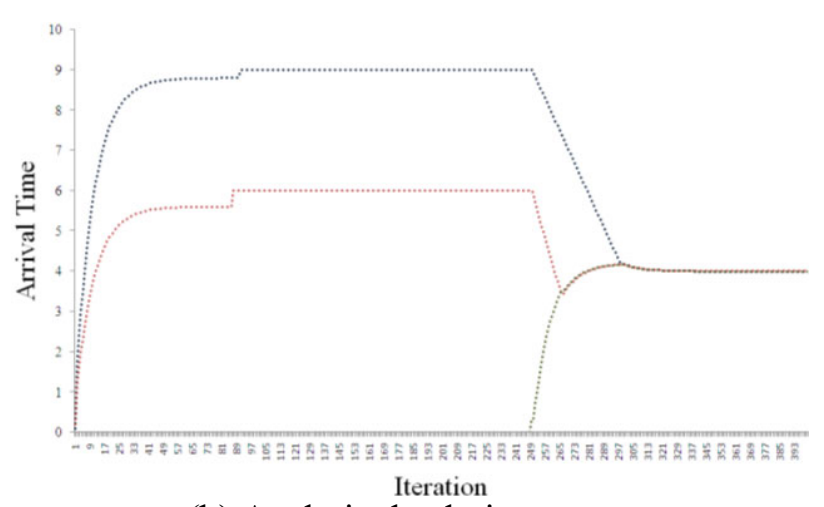

(b) Analytical solution

Figure 4: Arrival time trajectories

Transport time variations for each customer order through simulation time are shown in Figure 5. In each graph of transport time variation, the red solid-line indicates regular transport time and the black solid-line indicates the transport time adjusted by the distributed vehicle speed control. The unified controller provides a decision rule based on due-date deviation for each order so that it can adjust vehicle speed for both earliness and tardiness cases for the next iteration. Dynamics on vehicle speed are illustrated in Figure 5. In case of the first order as shown in Figure 5 (a), earliness occurs in interval [1,90] and [161, $400]$ along with tardiness interval [250,259]. For the second customer order, only earliness occurs within interval $[1,86]$ and $[251,400]$ as shown in Figure 5 (b). The third customer order also has only the earliness for due-date as shown in Figure 5 (c).

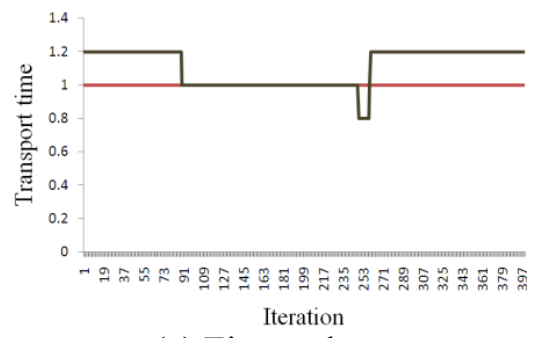

(a) First order

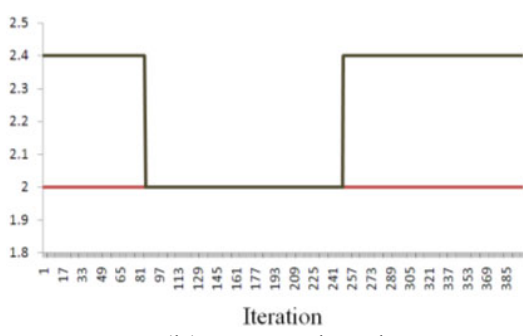

(b) Second order

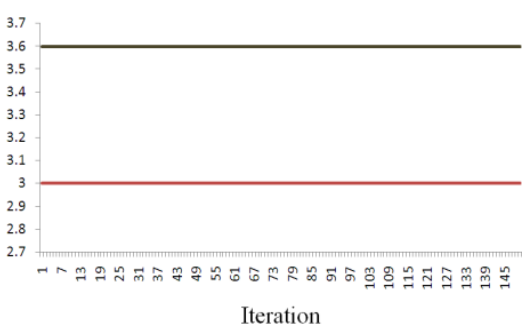

(c) Third order

Figure 5: Transport time variations

\section{PERFORMANCE OF UNIFIED MODEL}

The main objective of distributed arrival time and vehicle speed control is to minimize MSD by adjusting both vehicle routing schedules and vehicle speed simultaneously based on the customer order status such as earliness and tardiness. Along with MSD which is one of the representative performance measure for the customer service level, fuel efficiency of vehicle should be considered in terms of the transportation cost. Generally, fuel efficiency can be explained by two ways. The first one is fuel consumption which means the amount of fuel used per unit distance. The second one is fuel economy meaning the distance travelled per unit volume of fuel used. In this paper, we use fuel consumption for the vehicle fuel effi- 


\section{Lee and Prabhu}

ciency and it has a close relationship with vehicle speed as shown in Table 2 (West 1997). According to the experimental data from 1997, after the vehicle speed exceeds $55 \mathrm{MPH}$, the fuel consumption (MPG) shows tendency to gradually down.

Table 2: Fuel economy by speed studies, 1997 (MPG)

\begin{tabular}{|c|c|c|c|c|c|c|c|c|c|c|c|c|c|}
\hline MPH & 15 & 20 & 25 & 30 & 35 & 40 & 45 & 50 & 55 & 60 & 65 & 70 & 75 \\
\hline MPG & 24.4 & 27.9 & 30.5 & 31.7 & 31.2 & 31 & 31.6 & 32.4 & 32.4 & 31.4 & 29.2 & 26.8 & 24.8 \\
\hline
\end{tabular}

At first, the dynamics of the proposed distributed arrival time and vehicle speed control is tested in terms of customer service level which is explained by MSD and fuel consumption (MPG). Transportation data consisting of 8 customer orders, their geographical location and due-date information as shown in Table 3 is used. Regular, minimum and maximum vehicle speed limit are set as 55.00 MHP, 40.00 $\mathrm{MPH}$, and $70.00 \mathrm{MPH}$, respectively. By applying the speed controller, MSD is improved from 0.75 to 0.50 as shown in Table 4. Figure 6 (a) and (b) illustrate transportation routes on the Euclidean space when maximum speed limit is set as $40 \mathrm{MPH}$ and $70 \mathrm{MPH}$, respectively.

Table 3: Due-date and geographical information of customer orders

\begin{tabular}{|c|c|c|c|}
\hline Order No & X-coordinate (Mile) & Y-Coordinate (Mile) & Due-date (Hour) \\
\hline 1 & 35 & 35 & 4.18 \\
\hline 2 & 41 & 49 & 3.70 \\
\hline 3 & 35 & 17 & 3.66 \\
\hline 4 & 55 & 45 & 3.57 \\
\hline 5 & 55 & 20 & 2.88 \\
\hline 6 & 15 & 30 & 3.61 \\
\hline 7 & 25 & 30 & 1.97 \\
\hline 8 & 20 & 50 & 3.59 \\
\hline
\end{tabular}

Table 4: Results for (a) maximum speed limit $=40.00$, (b) maximum speed limit $=70.00$

\begin{tabular}{|c|c|c|c|c|c|c|c|c|c|}
\hline \multicolumn{2}{|c|}{ Route } & \multicolumn{2}{c|}{ Arrival Time (Hr) } & \multicolumn{2}{c|}{ Completion Time (Hr) } & \multicolumn{2}{c|}{ MPH } & \multicolumn{2}{c|}{ Due Dev (Hr) } \\
\hline (a) & (b) & (a) & (b) & (a) & (b) & (a) & (b) & (a) & (b) \\
\hline 5 & 7 & 0.19 & 0.63 & 1.55 & 1.54 & 40.00 & 40.00 & 1.34 & 0.44 \\
\hline 7 & 5 & 0.23 & 0.68 & 2.28 & 2.27 & 40.00 & 40.00 & -0.30 & 0.62 \\
\hline 3 & 4 & 0.27 & 0.70 & 2.66 & 2.85 & 40.00 & 40.00 & 1.01 & 0.73 \\
\hline 6 & 2 & 0.33 & 0.71 & 3.21 & 3.18 & 40.00 & 40.00 & 0.41 & 0.52 \\
\hline 8 & 8 & 0.36 & 0.71 & 3.60 & 3.60 & 40.00 & 50.24 & 0.00 & 0.00 \\
\hline 2 & 6 & 0.43 & 0.71 & 4.09 & 3.87 & 40.00 & 70.00 & -0.38 & -0.25 \\
\hline 4 & 1 & 0.46 & 0.75 & 4.42 & 4.18 & 40.00 & 64.63 & -0.84 & 0.00 \\
\hline 1 & 3 & 0.50 & 0.77 & 4.94 & 4.42 & 40.00 & 70.00 & -0.76 & -0.75 \\
\hline
\end{tabular}

Furthermore, the unified model is evaluated by using Solomon's VRP benchmark problems. We use 10 sets of problems which have 101 customer orders for each problem. Solomon's benchmark problems consist of customer demand data such as amount of demand, location of customers, and due-date. We use $\mathrm{X}$-coordinate and Y-coordinate to calculate regular transport time among customers by $55 \mathrm{MPH}$, and duedate by 24 hour units. To evaluate the relationship between MSD and fuel consumption, maximum speed limit is controlled differently for each simulation from $40 \mathrm{MPH}$ to $70 \mathrm{MPH}$. The resulting MSD values and performance changes are illustrated in Table 5. Compared to the results of DATC, about $10 \sim 40 \%$ improvement of MSD are achieved by the unified model except C203, C204, and RC204 problem sets as shown in Table 5. Except these cases, both average and minimum MSD values of the unified model are 


\section{Lee and Prabhu}

less than DATC results. For each problem set, we convert MSD values into relative values corresponding to the maximum MSD value of a problem set. After that, the range of these relative values including average value of problem sets are compared by increasing maximum speed limit from $40 \mathrm{MPH}$ to $70 \mathrm{MPH}$. Given the average values, they show downward trends as maximum vehicle speed limit increases as described in Figure 7.

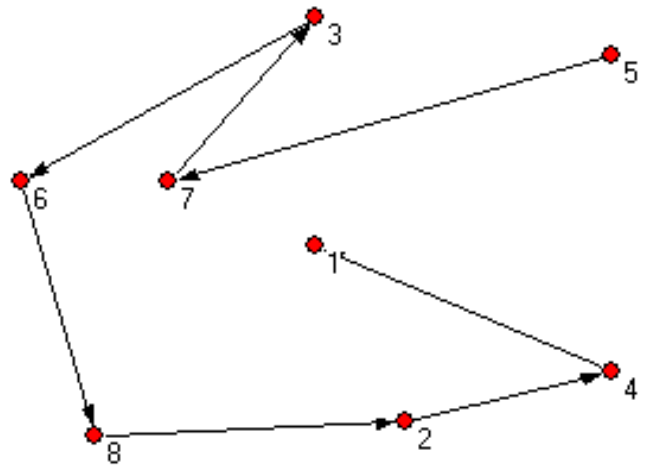

(a) $40 \mathrm{MPH}$ speed limit

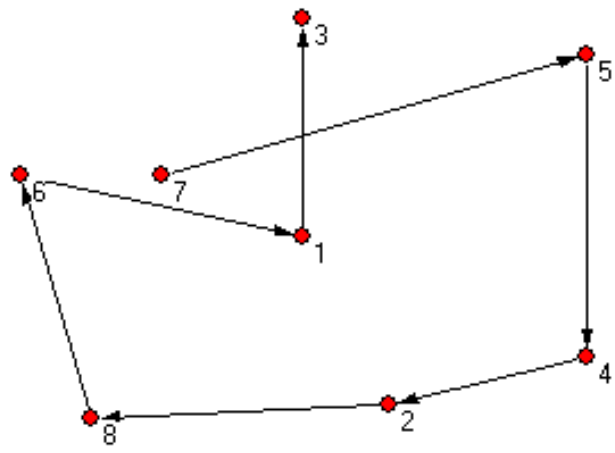

(b) $70 \mathrm{MPH}$ speed limit

Figure 6: Transportation routes

Table 5: MSD improvement by Solomon VRP data-set

\begin{tabular}{|c|c|c|c|c|c|c|}
\hline \multirow{2}{*}{$\begin{array}{c}\text { Problem } \\
\text { set }\end{array}$} & \multirow{2}{*}{$\begin{array}{c}\text { Number of } \\
\text { orders }\end{array}$} & DATC & \multicolumn{3}{|c|}{ Unified model } & Improvement for \\
\cline { 3 - 6 } & & Min & Average & Max & Min & Min value (\%) \\
\hline C201 & 101 & 0.0587 & 0.0439 & 0.0736 & 0.0588 & 25.10 \\
\hline C202 & 101 & 0.1776 & 0.1284 & 0.3006 & 0.2046 & 27.68 \\
\hline C203 & 101 & 0.7523 & 0.8755 & 2.5834 & 1.6249 & -16.38 \\
\hline C204 & 101 & 3.8757 & 4.7176 & 8.1220 & 6.7398 & -21.72 \\
\hline C101 & 101 & 0.1672 & 0.1208 & 0.2085 & 0.1641 & 27.78 \\
\hline C109 & 101 & 0.2166 & 0.1556 & 0.2941 & 0.2239 & 28.17 \\
\hline RC201 & 101 & 0.1672 & 0.1139 & 0.2121 & 0.1654 & 31.87 \\
\hline RC202 & 101 & 0.2792 & 0.1733 & 0.3620 & 0.2543 & 37.94 \\
\hline RC203 & 101 & 1.6389 & 1.4595 & 3.3896 & 2.4540 & 10.95 \\
\hline RC204 & 101 & 5.9079 & 6.4551 & 10.1023 & 8.5052 & -9.26 \\
\hline
\end{tabular}

A trade-off between average relative MSD and the rate of fuel consumption variations is illustrated in Figure 8. From $40 \mathrm{MPH}$ to $50 \mathrm{MPH}$, fuel consumption decreases gradually, and after the vehicle speed exceeds $55 \mathrm{MPH}$, it is on an increasing trend. Along with these experimental facts, average value of relative MSD also decreases gradually, and finally has about a 50\% value decreased from the $40 \mathrm{MPH}$ result. When maximum vehicle speed limit is set as about $66 \sim 67 \mathrm{MPH}$, a trade-off occurs in terms of a customer service level and a transportation cost level in these MPH interval. This fact can be used to develop a company's strategy for both improving customer service and reducing management costs. That is, according to a company's management strategy which puts more weight on customer service quality or management efficiency, economic guidelines for vehicle speed limit considering a vehicle routing schedule could be provided properly. 


\section{Lee and Prabhu}

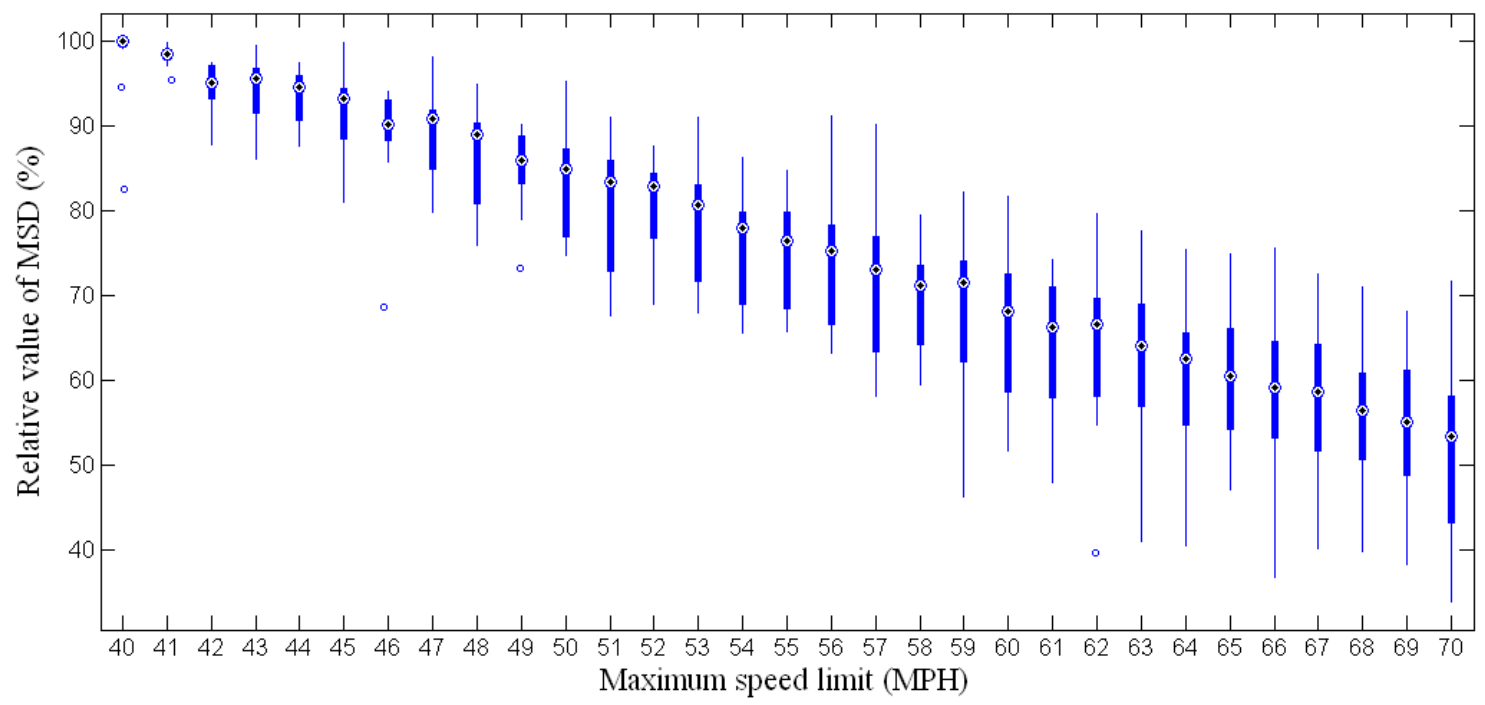

Figure 7: MSD variations

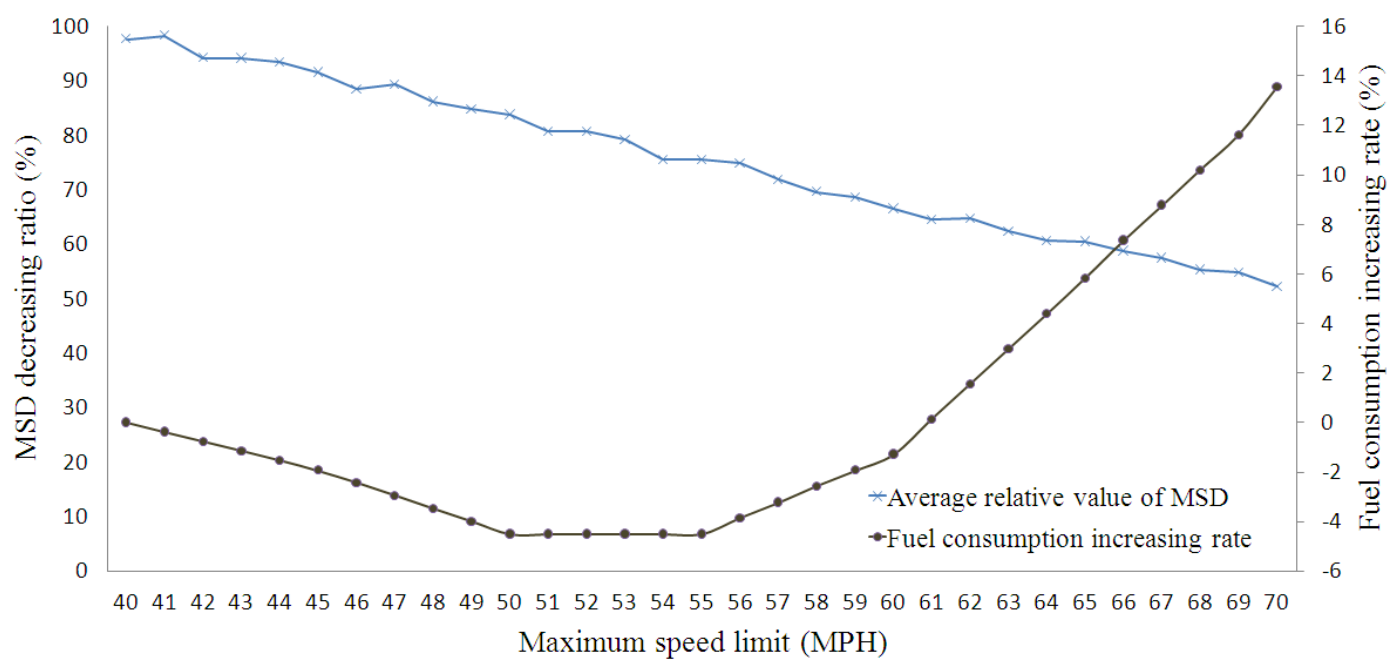

Figure 8: Comparison MSD with fuel consumption

\section{CONCLUSION}

In this paper, we proposed unified control algorithm for distributed arrival time and vehicle speed control based on control theoretic approach considering both the customer service level and transportation cost. The unified controller for vehicle routing scheduling and corresponding vehicle speed control was developed based on distributed arrival time control which is a feedback control-based scheduling approach for minimizing due-date deviation from customer demands. The comparison of analytical solutions and simulation results shows that core structure of the unified controller is operating properly for both feasible and infeasible due-date cases in terms of dynamics of vehicle routing scheduling environment. Using Solomon's VRP benchmark problem sets consisting of information for geographical location and customer due-date, the unified model was evaluated. Numerical experiments were conducted as varying maximum vehicle speed limits from $40 \mathrm{MPH}$ to $70 \mathrm{MPH}$ and it showed that average MSD improvement is $14.21 \%$ compared to the original DATC algorithm which has only a vehicle routing scheduling module. Furthermore, variation of maximum vehicle speed limits is accompanied by a trade-off between customer service quality and transportation costs. The relationship between these measures was shown by comparing MSD values and corresponding fuel consumption varying maximum vehicle speed limits. These facts can be 


\section{Lee and Prabhu}

used to build a guideline for a management strategy of a company or a logistics organization so that they can put economic weights on customer service quality or management efficiency according to their objectives.

\section{REFERENCES}

Cho, S., and V.V. Prabhu. 2002. Sliding mode dynamics in continuous feedback control for distributed discrete-event scheduling. Automatica 38 (9):1499-1515.

Cho, S., and V.V. Prabhu. 2007. Distributed adaptive control of production scheduling and machine capacity. Journal of Manufacturing Systems 26:65-74.

Lee, S., and V.V. Prabhu. 2009. Distributed arrival time control for vehicle routing problems with time windows. In Proceedings of 6th International Conference on Informatics in Control, Automation and Robotics, $246-251$.

Prabhu, V.V. 2009. Performance of real-time distributed arrival time control in heterarchical manufacturing systems. IIE Transactions 32(4):323-331.

Prabhu, V. V., and N.A. Duffie. 1999. Nonlinear dynamics in distributed arrival time control of heterarchical manufacturing systems. IEEE Transactions on Control Systems Technology 7(6):724-730.

Tamani, K., R. Boukezzoula, and G. Habchi. 2009. Hierarchical control of production flow based on capacity allocation for real-time scheduling of manufacturing systems. In Proceedings of the 14th IEEE international conference on emerging technologies \& factory automation, 519-526.

West, B.H., R.N. McGill, J.W. Hodgson, S.S. Sluder, and D.E. Smith. 1997 Development and verification of light-duty model emissions and fuel consumption values for traffic models. Federal Highway Administration Report, US Department of Transportation.

\section{AUTHOR BIOGRAPHIES}

SEOKGI LEE currently a Ph.D student in Industrial and Manufacturing Engineering at Penn State University, USA. His research interests include distributed control systems using control theory and discreteevents simulation for service engineering processes such as supply chain management and transportation. His email address is $\langle$ sul201@psu. edu $\rangle$.

VITTALDAS V. PRABHU received his Ph.D. in Mechanical Engineering from the University of Wisconsin-Madison, USA. He is currently a Professor in Industrial and Manufacturing Engineering at Penn State University, USA. His research interests include engineering of distributed control systems consisting of discrete-events, physical processes, and service processes. He teaches courses in controls, manufacturing, information, and service systems. His email address is <prabhulengr.psu. edu $>$. 